\title{
Synthesis and photophysical studies on triazole bridged dendrimers with phenothiazine as surface unit
}

\author{
Perumal Rajakumar,* Chinnadurai Satheeshkumar, and Mahalingam Ravivarma \\ Department of Organic Chemistry, University of Madras, Guindy Campus, Chennai 25, India \\ E-mail: perumalrajakumar@gmail.com
}

DOI: http://dx.doi.org/10.3998/ark.5550190.p008.375

\begin{abstract}
Synthesis and photophysical properties of some novel 1, 2, 3-triazole bridged phenothiazine dendrimers with enone and S-(-)-BINOL core is described.
\end{abstract}

Keywords: Dendrimer, phenothiazine, 1, 2, 3-triazole, click chemistry

\section{Introduction}

Dendrimers $^{1}$ are very unique type of macromolecule with hyperbranched and perfectly defined structure that have attracted much interest and an innovative area of research in supramolecular chemistry. Due to the special structure and unusual properties, dendrimers are utilized for a wide range of biomedical and material applications, such as antibacterial, ${ }^{2}$ drug delivery, ${ }^{3}$ lightharvesting ability, ${ }^{4}$ nonlinear optical (NLO), ${ }^{5}$ organic light-emitting diodes (OLEDs) ${ }^{6}$ and so on. Moreover, dendrimers with rigid structures can possibly be regularly assembled by packing on a plate without deformation of the molecule and are expected to expand the field of nanomaterials. ${ }^{7}$

Click chemistry coined by Sharpless, ${ }^{8}$ refers to a $\mathrm{Cu}(\mathrm{I})$ catalyzed Huisgen 1,3-dipolar cycloaddition of azides to alkynes, providing 1,4-disubtituted 1,2,3-triazole. The advantages of employing click chemistry are the excellent regioselectivity, tolerance of sensitive functional group, atom-economy, no protection-deprotection protocol, mild reaction conditions and excellent yields. Triazoles have interesting chemical properties, which include high aromatic stabilization and tolerance to acidic and basic as well as oxidative and reductive conditions. Moreover, 1,2,3-triazole derivatives are important materials in pharmaceuticals such as antiHIV ${ }^{9}$ antiviral, ${ }^{10}$ and antimicrobial. ${ }^{11}$ In addition, triazole derivatives play a pivotal role in the field of supramolecular assemblies. ${ }^{12}$ Furthermore, synthesis of dendrimers using click chemistry has received much attention during recent times due to their interesting biological applications. ${ }^{13}$ 

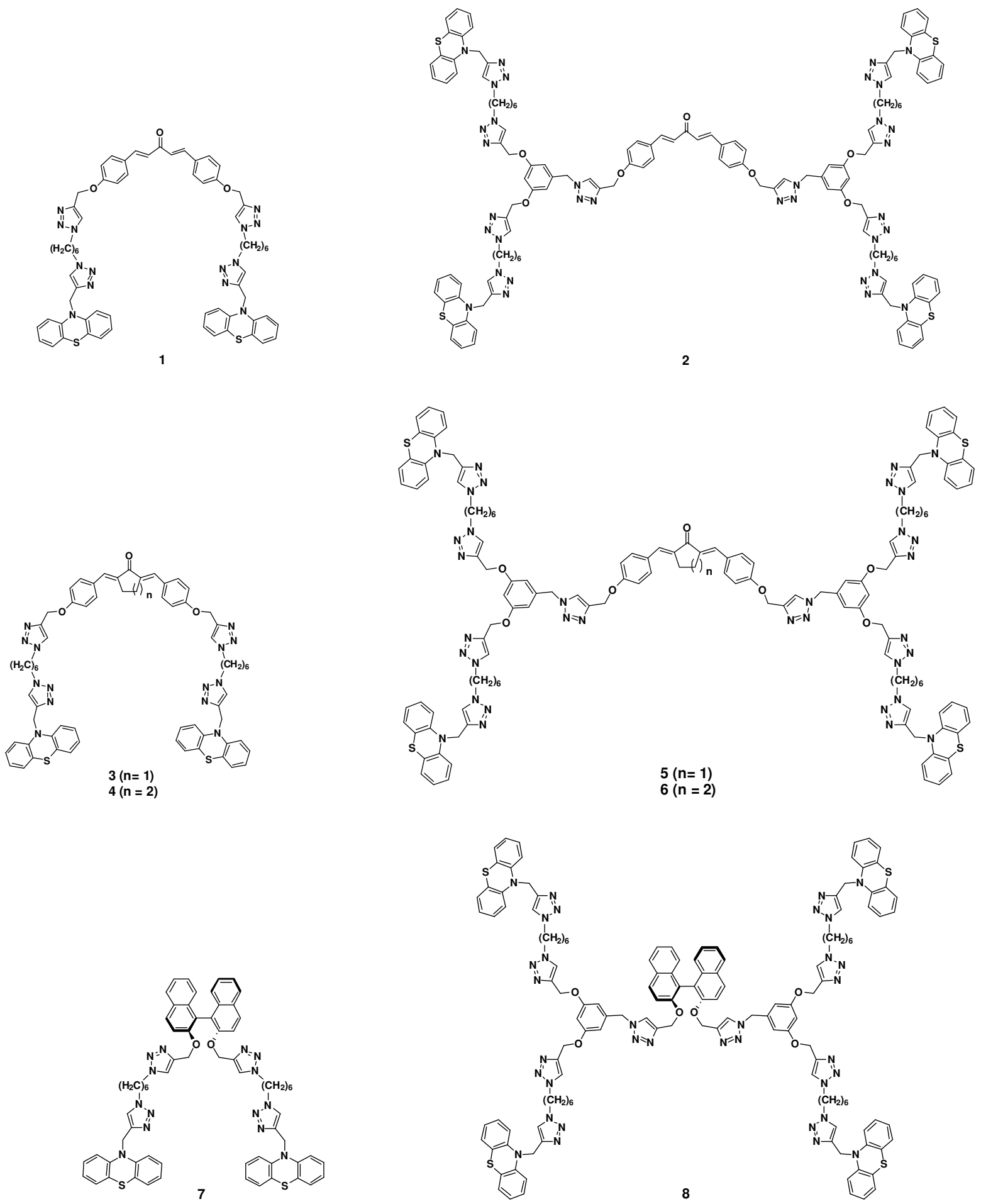

Figure 1. Molecular structure of phenothiazine dendrimers 1-8.

Recently, we have reported the synthesis of dendrimer with dimethyl isophthalate, ${ }^{14}$ pyrrolidine, ${ }^{15}$ quinoline ${ }^{16}$ and pyreno-chalcone ${ }^{17}$ as surface groups with $1,2,3$-triazole as 
bridging unit. Phenothiazine belongs to an important class of tricyclic nitrogen-sulphur heterocyclic compound. phenothiazine-based dendrimers exhibit remarkable optical and electrochemical properties. ${ }^{18}$ Further, the phenothiazine heterocyclic system shows antimicrobial, anticancer, antiviral, anticonvulsant and antimalarial activity. ${ }^{19}$ The dendrimers with S-(-)BINOL core could exhibit chiroptical, photophysical properties, ${ }^{20}$ and also used as enantioselective Lewis acid catalysts. ${ }^{21}$ In connection with this, the present investigation deals with the synthesis and photophysical properties of some novel triazole bridged dendrimers 1-8 (Figure 1) bearing phenothiazine as surface group by convergent methodology.

\section{Results and Discussion}

The bifunctional 1-azido-6-bromohexane $\mathbf{1 0}$ was synthesized by the reaction of 1,6dibromohexane with one equivalent of $\mathrm{NaN}_{3}$ in a mixture of acetone and water $(9: 1)$ at $60{ }^{\circ} \mathrm{C}$. Further, reaction of $N$-propargyl phenothiazine ${ }^{22} \mathbf{9}$ with the azide $\mathbf{1 0}$ under click reaction conditions of $\mathrm{CuSO}_{4} .5 \mathrm{H}_{2} \mathrm{O}(5 \mathrm{~mol} \%)$ and sodium ascorbate $(10 \mathrm{~mol} \%)$ in a mixture of THF and water (1:1) at room temperature for $10 \mathrm{~h}$ gave the bromo compound $\mathbf{1 1}$ in $90 \%$ yield. The bromide 11 was converted to the azide 12 in $91 \%$ yield using $\mathrm{NaN}_{3}$. Reaction of 3,5bis(propargyloxy)benzyl chloride ${ }^{16} \mathbf{1 3}$ with 2.1 equiv. of azide dendron $\mathbf{1 2}$ under click chemistry conditions afforded the first generation dendritic chloride $\left(\mathrm{G}_{1}-\mathrm{Cl}\right) 14$ in $89 \%$ yield. The dendritic chloride 14 with $\mathrm{NaN}_{3}$ in DMF at $60{ }^{\circ} \mathrm{C}$ afforded the first generation dendritic azide $\left(\mathrm{G}_{1}-\mathrm{N}_{3}\right) \mathbf{1 5}$ in $87 \%$ yield (see Scheme 1).

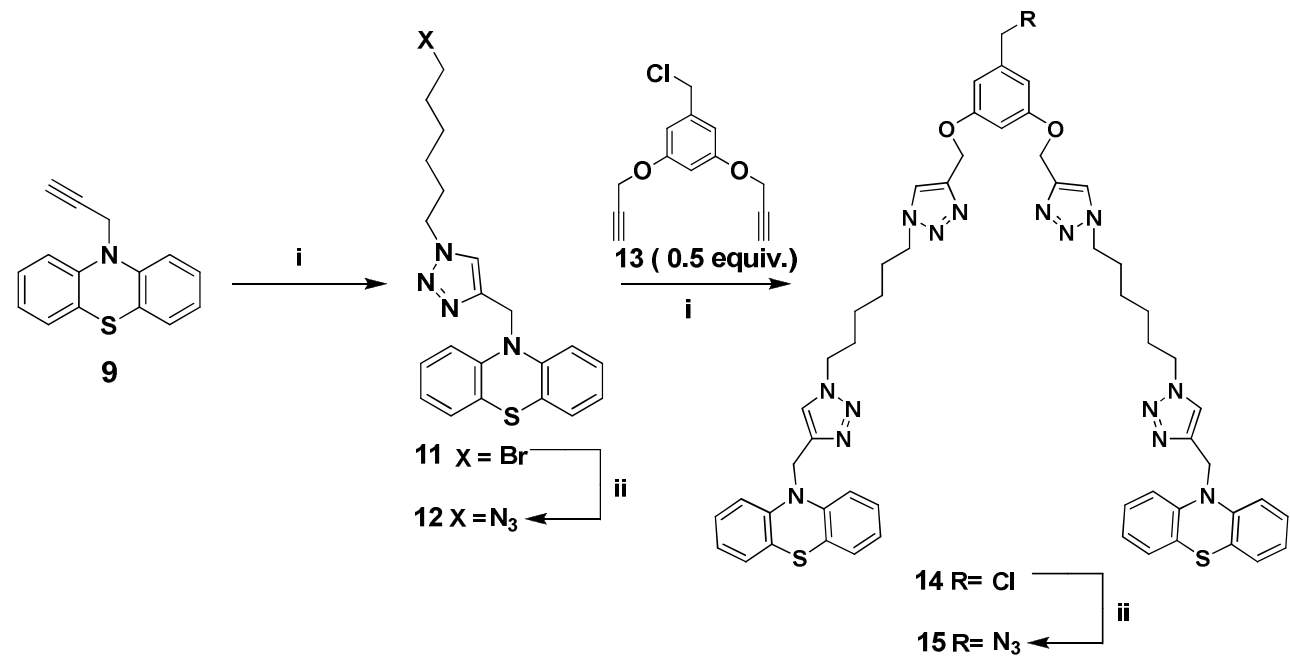

Scheme 1. Reagents and conditions: (i) $\mathrm{Br}-\left(\mathrm{CH}_{2}\right)_{6}-\mathrm{N}_{3}$, (10) (1 equiv.), $\mathrm{CuSO}_{4} .5 \mathrm{H}_{2} \mathrm{O}$ (5 mol \%), sodium ascorbate (10 mol \%), THF: $\mathrm{H}_{2} \mathrm{O}(1: 1, \mathrm{v} / \mathrm{v}), \mathrm{rt}, 10 \mathrm{~h}, \mathbf{1 1}(90 \%)$ and 14 (89\%). (ii) $\mathrm{NaN}_{3}$, DMF, $10 \mathrm{~h}, 60{ }^{\circ} \mathrm{C}, 12(91 \%)$ and $15(87 \%)$. 
The ${ }^{1} \mathrm{H}$ NMR spectrum of compound $\mathbf{1 5}$ displayed the ten methylene protons adjacent to the nitrogen atom of triazole ring and azide functionality at $\delta 4.11-4.2$, and the rest of the methylene protons of the hexyl unit appear as two set of multiplets eight proton each at $\delta 1.11-1.18$ and 1.56-1.85 and singlets at $\delta 5.08$ and 5.10 for $\mathrm{N}-\mathrm{CH}_{2}$ - adjacent to phenothiazine and $O-\mathrm{CH}_{2}$ protons respectively in addition to the other aliphatic and aromatic proton signals. The ${ }^{13} \mathrm{C} \mathrm{NMR}$ spectrum of dendritic azide 15 showed $N-\mathrm{CH}_{2}$ - and $O-\mathrm{CH}_{2}$ - carbon signals at $\delta 54.5$ and 61.9 in addition to the aliphatic and aromatic carbon signals. The ESI mass spectrum of $\mathbf{1 5}$ showed molecular ion peak $\mathrm{m} / z 1052(\mathrm{M}+\mathrm{H})^{+}$. The molecular formula of $\mathbf{1 5}$ was further confirmed from analytical data.

The core units bis(propargyloxy)dienone 19, bis(propargyloxy)pentanone 20, bis(propargyloxy)hexanone ${ }^{23} \mathbf{2 1}$ and bis(propargyloxy)S-(-) BINOL 22 were obtained in 63\%, $82 \%, 76 \%$ and $78 \%$ yields respectively by the $O$-alkylation of $16,17,18$ and S-(-) BINOL with 2.1 equiv. of propargyl bromide in the presence of $\mathrm{K}_{2} \mathrm{CO}_{3}$ in DMF (Scheme 2).
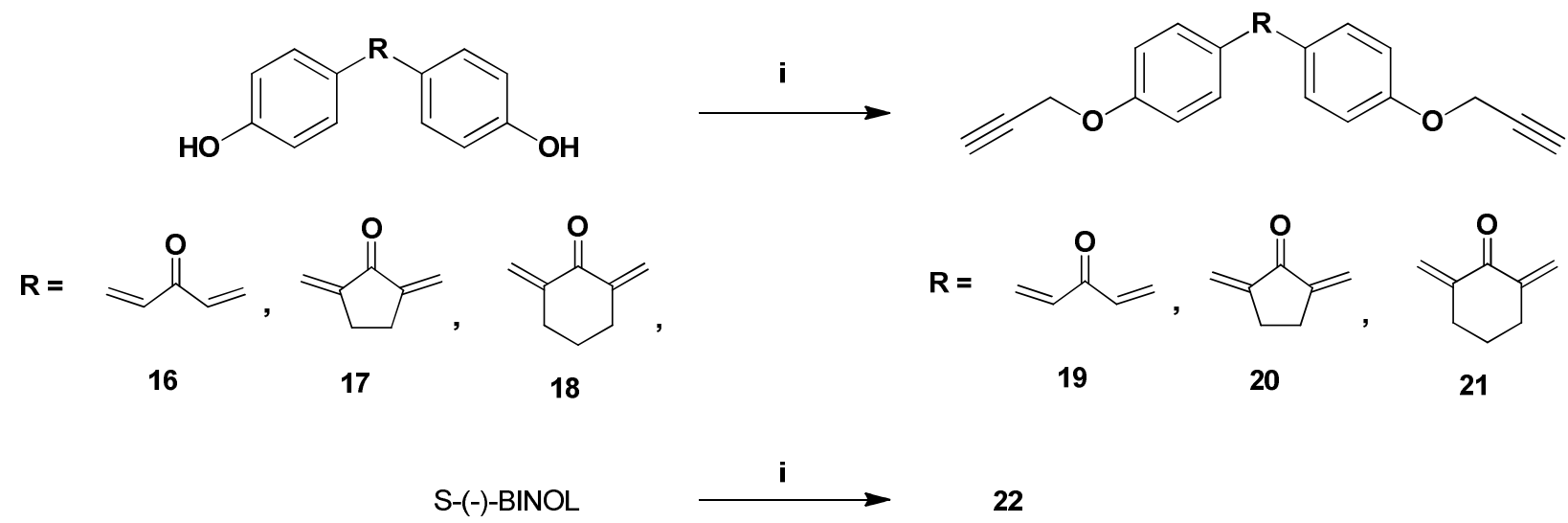

Scheme 2. Reagents and conditions: (i) Propargyl bromide (2.1 equiv.), $\mathrm{K}_{2} \mathrm{CO}_{3}$, DMF, $60{ }^{\circ} \mathrm{C}, 24$ h, $19(63 \%), 20(82 \%), 21(76 \%)$ and $22(78 \%)$.

The synthetic pathway leading to the enone and S-(-)-BINOL based zeroth generation dendrimers with phenothiazine surface group is shown in Scheme 3. Reaction of bispropargyloxy core unit 19-21 and 22 with phenothiazine dendritic azide 12 under click reaction conditions afforded 1, 2, 3-triazole bridged phenothiazine dendrimers 1, 3, 4 and 7 in good yields (see Scheme 3). The ${ }^{1} \mathrm{H}$ NMR spectrum of dendrimer 4 displayed singlets at $\delta 5.20$ and 5.25 for the $\mathrm{N}$-methylene and $\mathrm{O}$-methylene protons, in addition to the signals for aliphatic and aromatic protons. The ${ }^{13} \mathrm{C}$ NMR spectrum of dendrimer 4 displayed $N$-methylene and $O$ - methylene carbons at $\delta 49.8$ and 61.8 respectively, the carbonyl carbon appeared at $\delta 189.7$, in addition to the signals for aliphatic and aromatic carbons. The appearance of molecular ion peak at $\mathrm{m} / z$ 1215.6 $(\mathrm{M}+\mathrm{Na})^{+}$in mass spectrum of the dendrimer $\mathbf{4}$ also confirmed the structure. Similarly, the structure of the dendrimers $\mathbf{1}, \mathbf{3}$ and $\mathbf{7}$ was confirmed from spectral and analytical data. 

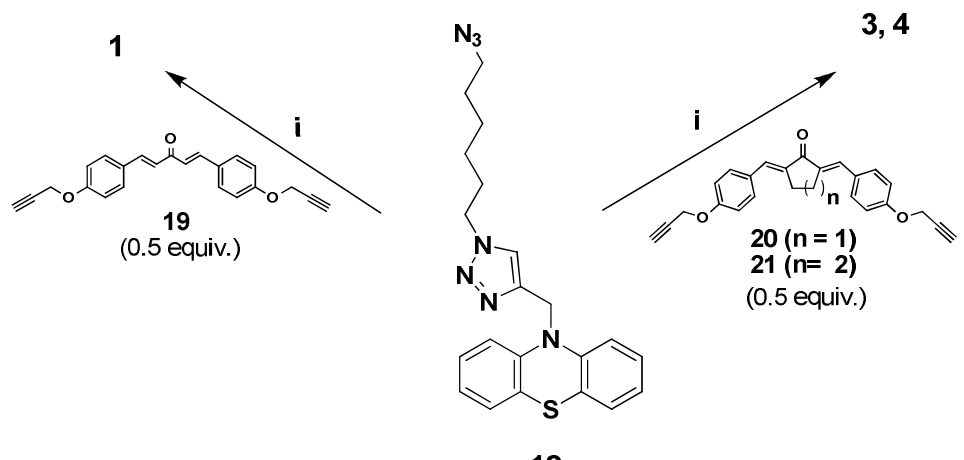

12

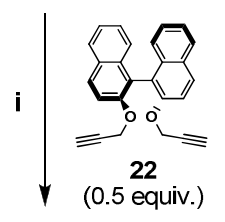

7

Scheme 3. Reagents and conditions: (i) $\mathrm{CuSO}_{4} .5 \mathrm{H}_{2} \mathrm{O}(5 \mathrm{~mol} \%)$, sodium ascorbate (10 mol \%), THF: $\mathrm{H}_{2} \mathrm{O}(1: 1, \mathrm{v} / \mathrm{v}), \mathrm{rt}, 10 \mathrm{~h}, \mathbf{1}(86 \%), \mathbf{3}(84 \%), \mathbf{4}(85 \%)$ and 7 (92\%).

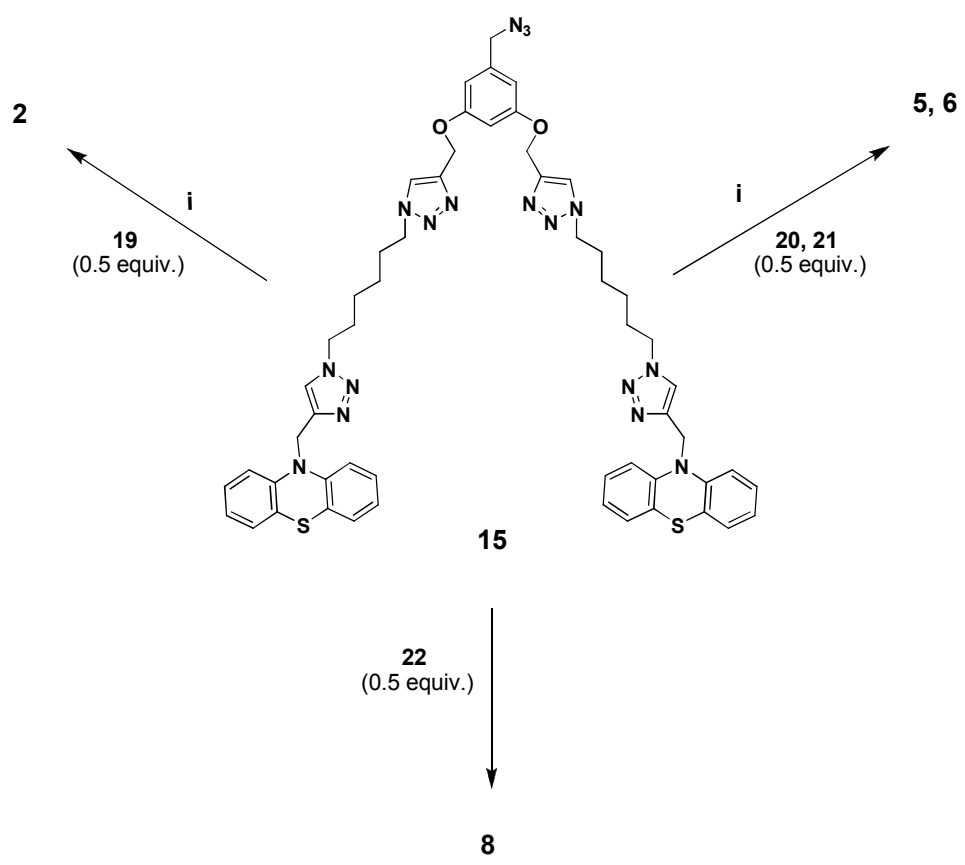

Scheme 4. Reagents and conditions: (i) $\mathrm{CuSO}_{4} .5 \mathrm{H}_{2} \mathrm{O}(5 \mathrm{~mol} \%)$, sodium ascorbate (10 mol \%), THF: $\mathrm{H}_{2} \mathrm{O}(1: 1, \mathrm{v} / \mathrm{v}), \mathrm{rt}, 10 \mathrm{~h}, \mathbf{2}(84 \%), \mathbf{5}(80 \%), \mathbf{6}(86 \%)$ and $\mathbf{8}(83 \%)$.

Similar technique was adopted to synthesize the first generation dendrimers. Reaction of the bis-propargyloxy core moieties 19-22 with 2.0 equiv. of dendritic azide $\left(\mathrm{G}_{1}-\mathrm{N}_{3}\right) \mathbf{1 5}$ in the 
presence of the $\mathrm{Cu}(\mathrm{I})$-catalyzed Huisgen click reaction conditions generated the first generation dendrimers $\left(\mathrm{G}_{1}\right)$ 2, 5, 6 and $\mathbf{8}$ in 84\%, 80\%, 86\% and 83\% yields, respectively (see Scheme 4). In the ${ }^{1} \mathrm{H}$ NMR spectrum, the chiral phenothiazine dendrimer 8 showed singlets at $\delta 5.07$ for $N$ methylene and at $\delta 5.17$ for $O$-methylene protons, in addition to the signals for aliphatic and aromatic protons. The ${ }^{13} \mathrm{C}$ NMR spectrum of dendrimer 8 displayed $N$-methylene and $O$ methylene carbons at $\delta 50.0,53.7,61.9$ and 63.9 in addition to the signals for aliphatic and aromatic carbons. In the MALDI-TOF mass spectrum of $\mathbf{8}$, the molecular ion peak appeared at $\mathrm{m} / \mathrm{z} 2466(\mathrm{M})^{+}$and the structure of the dendrimer $\mathbf{8}$ was further confirmed from analytical data. Similarly, the structure of the dendrimers 2,5 and $\mathbf{6}$ was confirmed from spectral and analytical data.

\section{Photophysical studies}

The photophysical property of phenothiazine dendrimers 1-8 is listed in Table 1. Figure 2a show the absorption spectra of dendrimers 1-8 in DMF.. There is a broad absorption band at 284-386 $\mathrm{nm}$ due to the presence of triazole and phenothiazine units. In fact this observation suggests that both the chromophores viz triazole and phenothiazine could be responsible for the broad absorption bands for the dendrimers 1-8. Molar extinction coefficients of the absorption bands at a given concentration varies as the generation of the dendrimer increases. The absorbance in the UV spectrum is probably controlled by the number of phenothiazine and triazole units.

Table 1. Photophysical data for dendrimers 1-8 in DMF $\left(1 \times 10^{-5} \mathrm{~mol} / \mathrm{L}\right)$

\begin{tabular}{ccccc}
\hline Dendrimers & $\begin{array}{c}\lambda_{\text {abs } \max } \\
(\mathrm{nm})\end{array}$ & $\begin{array}{c}\varepsilon \times 10^{-5} \\
\mathrm{M}^{-1} \mathrm{~cm}^{-1}\end{array}$ & $\begin{array}{c}\lambda_{\mathrm{em}} \max \\
(\mathrm{nm})\end{array}$ & $\Phi_{\mathrm{F}}$ \\
\hline $\mathbf{1}$ & 359 & 1.55 & 445 & 0.0017 \\
$\mathbf{2}$ & 321,358 & $0.96,0.91$ & 440,520 & 0.0036 \\
$\mathbf{3}$ & 386 & 0.56 & 433,462 & 0.0050 \\
$\mathbf{4}$ & 358 & 0.92 & 438 & 0.0032 \\
$\mathbf{5}$ & 385 & 0.65 & 439 & 0.0143 \\
$\mathbf{6}$ & 321,357 & $0.52,0.61$ & 431 & 0.0070 \\
$\mathbf{7}$ & 297,324 & $0.26,0.21$ & 433 & 0.0778 \\
$\mathbf{8}$ & 312 & 0.34 & 437 & 0.1148 \\
\hline
\end{tabular}


a)

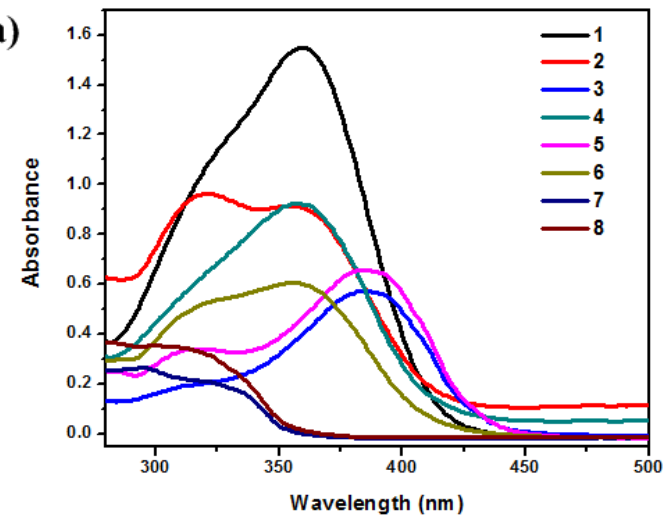

b)

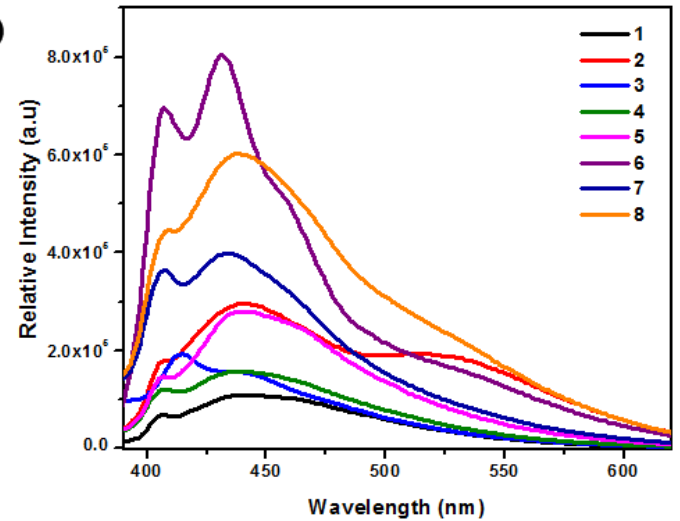

Figure 2. (a) Absorption spectra of dendrimers 1-8 in DMF $\left(1 \times 10^{-5} \mathrm{~mol} / \mathrm{L}\right)$. (b) Fluorescence spectra of dendrimers 1-8 in DMF $\left(1 \times 10^{-5} \mathrm{~mol} / \mathrm{L}\right)$.

Figures $2 \mathrm{~b}$ shows the fluorescence spectra of dendrimers 1-8 in DMF and the fluorescence parameters for all the dendrimers are presented in Table 1. As shown in Figure 3, on excitation at $360 \mathrm{~nm}$, the dendrimers 1-8 give emission band in range at 431-520 $\mathrm{nm}$ due to the triazole and phenothiazine moieties. The fluorescence intensity of the dendrimers increases as the generation increases, which are consistent with the increased number of both fluorophoric triazole and phenothiazine units, otherwise known as multivalency effect in dendrimer chemistry. The fluorescence quantum yields $\Phi_{\mathrm{f}}$ of dendrimers 1-8 have been measured in DMF using quinine sulphate in $0.1 \mathrm{~N} \mathrm{H}_{2} \mathrm{SO}_{4}$ as the standard. The quantum yields of dendrimers 1-8 are listed in Table 1. Thus as the generation of the dendrimer increases, the quantum yield also increases.

\section{Conclusions}

In conclusion, we have synthesized various dendritic architectures with phenothiazine surface group and 1,2,3-triazole as bridging unit through click reaction by convergent approach in good yields. The dendrimers reported herein possesses biologically active enone core and phenothiazine surface group. Synthesis of other such phenothiazine dendrimers and their biological activity are under way.

\section{Experimental Section}

General. All the melting points reported were uncorrected and are determined using Toshniwal melting point apparatus by open capillary tube method. The ${ }^{1} \mathrm{H}$ and ${ }^{13} \mathrm{C}$ NMR spectra were recorded on Bruker $300 \mathrm{MHz}$ spectrometer. The chemical shifts are reported in ppm $(\delta)$ with TMS as an internal standard and coupling constant $(J)$ are expressed in Hz. MALDI-TOF mass 
spectra on Voyager-DE PRO mass spectrometer using a $\alpha$-cyano-4-hydroxy cinnamic acid (CHCA) matrix and ESI-PerkinElmer Sciex, API 3000 mass spectrometer. The QTOF-MS spectra were recorded on Xevo G2-S QT mass spectrometer. Elemental analyses were performed on a Perkin-Elmer 240B elemental analyzer. TLC was performed either on glass plates coated with silica gel-G (ACME) of about $0.25 \mathrm{~mm}$ thickness and visualized with iodine or on pre-coated plastic sheets (POLYGRAM ${ }^{\circledR}$ SIL G/U $\mathrm{U}_{254}$ ) and detected under UV light. Column chromatography was carried out with silica gel (ACME, 60-120 and 100-200 mesh).

General procedure for the synthesis of 1,2,3-triazole using click chemistry. A mixture of azide (1.0 equiv.), alkyne (0.5 equiv.), $\mathrm{CuSO}_{4} .5 \mathrm{H}_{2} \mathrm{O}(5 \mathrm{~mol} \%)$ and $\mathrm{NaAsc}$. $(10 \mathrm{~mol} \%)$ in a mixture of THF and water (1:1) was stirred for $10 \mathrm{~h}$ at room temperature. The residue obtained after evaporation of the solvent was washed thoroughly with water and dissolved in $\mathrm{CHCl}_{3}(150$ $\mathrm{mL})$. The organic layer was washed with water $(2 \times 100 \mathrm{~mL})$ and brine $(1 \times 150 \mathrm{~mL})$ and dried $\left(\mathrm{Na}_{2} \mathrm{SO}_{4}\right)$ and evaporated to give the crude triazole, which was purified by column chromatography $\left(\mathrm{SiO}_{2}\right)$.

Dendrimer $1\left(\mathbf{G}_{\mathbf{0}}\right)$. Yield $86 \%$, mp $78{ }^{\circ} \mathrm{C} .{ }^{1} \mathrm{H}$ NMR $\left(300 \mathrm{MHz}, \mathrm{CDCl}_{3}\right): \delta_{\mathrm{H}} 1.20-1.25(\mathrm{~m}, 8 \mathrm{H})$, 1.77-1.86 (m, 8H), $4.24(\mathrm{t}, 4 \mathrm{H}, J=6.9 \mathrm{~Hz}), 4.30(\mathrm{t}, 4 \mathrm{H}, J=7.2 \mathrm{~Hz}), 5.20(\mathrm{~s}, 4 \mathrm{H}), 5.26(\mathrm{~s}, 4 \mathrm{H})$, $6.76(\mathrm{~d}, 4 \mathrm{H}, J=8.1 \mathrm{~Hz}), 6.86-6.93(\mathrm{~m}, 4 \mathrm{H}), 6.98-7.07(\mathrm{~m}, 8 \mathrm{H}), 7.12(\mathrm{~d}, 4 \mathrm{H}, J=7.8 \mathrm{~Hz}), 7.26(\mathrm{~s}$, $4 \mathrm{H}), 7.55(\mathrm{~s}, 2 \mathrm{H}), 7.58(\mathrm{~s}, 4 \mathrm{H}), 7.69(\mathrm{~d}, 2 \mathrm{H}, J=15.9 \mathrm{~Hz}) .{ }^{13} \mathrm{C} \mathrm{NMR}\left(75 \mathrm{MHz}, \mathrm{CDCl}_{3}\right): \delta_{\mathrm{C}} 25.6$, 25.7, 29.8, 29.9, 44.9, 50.0, 50.1, 62.1, 115.2, 115.3, 122.3, 122.6, 122.8, 123.8, 123.9, 127.2, 127.3, 128.1, 130.1, 142.5, 143.7, 144.3, 144.9, 160.1, 188.8. ESI-MS: $m / z=1154(\mathrm{M}+\mathrm{H})^{+}$. Anal. calcd. for $\mathrm{C}_{65} \mathrm{H}_{64} \mathrm{~N}_{14} \mathrm{O}_{3} \mathrm{~S}_{2}: \mathrm{C}, 67.68 ; \mathrm{H}, 5.59 ; \mathrm{N}, 17.00 \%$. Found: $\mathrm{C}, 67.54 ; \mathrm{H}, 5.67 ; \mathrm{N}$, $17.10 \%$.

Dendrimer $2\left(\mathbf{G}_{\mathbf{1}}\right)$. Yield $84 \%, \mathrm{mp} 100{ }^{\circ} \mathrm{C} .{ }^{1} \mathrm{H}$ NMR $\left(300 \mathrm{MHz}, \mathrm{CDCl}_{3}\right): \delta_{\mathrm{H}} 1.17-1.26(\mathrm{~m}, 16 \mathrm{H})$, $1.74-1.83(\mathrm{~m}, 16 \mathrm{H}), 4.21(\mathrm{t}, 8 \mathrm{H}, J=6.9 \mathrm{~Hz}), 4.24(\mathrm{t}, 8 \mathrm{H}, J=6.9 \mathrm{~Hz}), 5.10(\mathrm{~s}, 8 \mathrm{H}), 5.17(\mathrm{~s}, 8 \mathrm{H})$, $5.20(\mathrm{~s}, 4 \mathrm{H}), 5.42(\mathrm{~s}, 4 \mathrm{H}), 6.49(\mathrm{~d}, 4 \mathrm{H}, J=1.8 \mathrm{~Hz}), 6.61(\mathrm{t}, 2 \mathrm{H}, J=2.1 \mathrm{~Hz}), 6.75(\mathrm{~d}, 8 \mathrm{H}, J=8.1$ $\mathrm{Hz}), 6.84-6.90(\mathrm{~m}, 12 \mathrm{H}), 6.95-7.04(\mathrm{~m}, 16 \mathrm{H}), 7.08(\mathrm{~d}, 8 \mathrm{H}, J=7.5 \mathrm{~Hz}), 7.51(\mathrm{~s}, 2 \mathrm{H}), 7.56(\mathrm{~s}, 4 \mathrm{H})$, $7.61(\mathrm{~s}, 4 \mathrm{H}) .{ }^{13} \mathrm{C} \mathrm{NMR}\left(75 \mathrm{MHz}, \mathrm{CDCl}_{3}\right): \delta_{\mathrm{C}} 25.5,25.6,29.7,29.8,44.9,49.9,50.0,54.0,61.9$, 102.0, 107.4, 115.2, 122.3, 122.8, 123.1, 123.7, 123.8, 127.1, 127.3, 128.1, 130.1, 136.8, 142.4, 143.4, 143.9, 144.2, 144.7, 159.8, 160.0, 188.7. QTOF-MS: $m / z=2445.98\left(\mathrm{M}^{+}\right)$. Anal. calcd. for $\mathrm{C}_{133} \mathrm{H}_{132} \mathrm{~N}_{34} \mathrm{O}_{7} \mathrm{~S}_{4}: \mathrm{C}, 65.28 ; \mathrm{H}, 5.44 ; \mathrm{N}, 19.46 \%$. Found: $\mathrm{C}, 65.42 ; \mathrm{H}, 5.53 ; \mathrm{N}, 19.38 \%$.

Dendrimer $3\left(\mathbf{G}_{\mathbf{0}}\right)$. Yield $84 \%$, mp $198{ }^{\circ} \mathrm{C} .{ }^{1} \mathrm{H}$ NMR $\left(300 \mathrm{MHz}, \mathrm{CDCl}_{3}\right)$ : $\delta_{\mathrm{H}} 1.19-1.33(\mathrm{~m}, 8 \mathrm{H})$, $1.77-1.88(\mathrm{~m}, 8 \mathrm{H}), 3.06(\mathrm{~s}, 4 \mathrm{H}), 4.23(\mathrm{t}, 4 \mathrm{H}, J=6.9 \mathrm{~Hz}), 4.29(\mathrm{t}, 4 \mathrm{H}, J=6.9 \mathrm{~Hz}), 5.19(\mathrm{~s}, 4 \mathrm{H})$, $5.26(\mathrm{~s}, 4 \mathrm{H}), 6.76(\mathrm{~d}, 4 \mathrm{H}, J=8.1 \mathrm{~Hz}), 6.88(\mathrm{t}, 4 \mathrm{H}, J=7.5 \mathrm{~Hz}), 7.01-7.06(\mathrm{~m}, 8 \mathrm{H}), 7.09-7.12(\mathrm{~m}$, $4 \mathrm{H}), 7.27$ (s, 2H), 7.54-7.57 (m, 8H). ${ }^{13} \mathrm{C} \mathrm{NMR}\left(75 \mathrm{MHz}, \mathrm{CDCl}_{3}\right): \delta_{\mathrm{C}} 25.6,25.7,26.5,29.8,29.9$, 44.9, 50.0, 50.1, 62.1, 115.1, 115.3, 122.3, 122.6, 122.8, 123.9, 127.2, 127.3, 129.3, 132.5, 133.1, 135.6, 143.8, 144.3, 144.9, 159.1, 196.2. ESI-MS: $m / z=1179.4(\mathrm{M}+\mathrm{H})^{+}$. Anal. calcd. for $\mathrm{C}_{67} \mathrm{H}_{66} \mathrm{~N}_{14} \mathrm{O}_{3} \mathrm{~S}_{2}$ : C, 68.23; $\mathrm{H}, 5.64 ; \mathrm{N}, 16.63 \%$. Found: $\mathrm{C}, 68.48 ; \mathrm{H}, 5.53 ; \mathrm{N}, 16.72 \%$.

Dendrimer $4\left(\mathbf{G}_{0}\right)$. Yield 85\%, mp 172-174 ${ }^{\circ} \mathrm{C} .{ }^{1} \mathrm{H}$ NMR $\left(300 \mathrm{MHz}, \mathrm{CDCl}_{3}\right): \delta_{\mathrm{H}} 1.20-1.25(\mathrm{~m}$, $8 \mathrm{H}), 1.78-1.86(\mathrm{~m}, 10 \mathrm{H}), 2.91(\mathrm{t}, 4 \mathrm{H}, J=5.1 \mathrm{~Hz}), 4.24(\mathrm{t}, 4 \mathrm{H}, J=7.2 \mathrm{~Hz}), 4.30(\mathrm{t}, 4 \mathrm{H}, J=7.2$ 
$\mathrm{Hz}), 5.20(\mathrm{~s}, 4 \mathrm{H}), 5.25(\mathrm{~s}, 4 \mathrm{H}), 6.76(\mathrm{~d}, 4 \mathrm{H}, J=8.1 \mathrm{~Hz}), 6.89(\mathrm{t}, 4 \mathrm{H}, J=7.5 \mathrm{~Hz}), 7.01-7.07(\mathrm{~m}$, $8 \mathrm{H}), 7.12(\mathrm{~d}, 4 \mathrm{H}, J=7.5 \mathrm{~Hz}), 7.27(\mathrm{~s}, 2 \mathrm{H}), 7.44(\mathrm{~d}, 4 \mathrm{H}, J=8.7 \mathrm{~Hz}), 7.57(\mathrm{~s}, 2 \mathrm{H}), 7.74(\mathrm{~s}, 2 \mathrm{H})$. ${ }^{13} \mathrm{C}$ NMR $\left(75 \mathrm{MHz}, \mathrm{CDCl}_{3}\right): \delta_{\mathrm{C}} 22.7,25.3,25.4,28.2,29.5,29.6,44.6,49.7,49.8,61.8,114.4$, 114.9, 122.0, 122.2, 122.5, 123.6, 126.9, 127.0, 128.9, 131.9, 134.3, 136.0, 143.6, 143.9, 144.6, 158.2, 189.7. ESI-MS: $m / z=1193.4(\mathrm{M}+\mathrm{H})^{+}, m / z=1215.6(\mathrm{M}+\mathrm{Na})^{+}$. Anal. calcd. for $\mathrm{C}_{68} \mathrm{H}_{68} \mathrm{~N}_{14} \mathrm{O}_{3} \mathrm{~S}_{2}$ : C, 68.43; H, 5.74; N, 16.43\%. Found: C, 68.64; H, 5.87; N, $16.52 \%$.

Dendrimer $5\left(\mathbf{G}_{1}\right)$. Yield $80 \%$, mp 142-144 ${ }^{\circ} \mathrm{C} .{ }^{1} \mathrm{H}$ NMR $\left(300 \mathrm{MHz}, \mathrm{DMSO}-\mathrm{d}_{6}\right): \delta_{\mathrm{H}} 1.10-1.13$ (m, 16H), 1.67-1.74 (m, 16H), $3.01(\mathrm{~s}, 4 \mathrm{H}), 4.23-4.31(\mathrm{~m}, 16 \mathrm{H}), 5.11(\mathrm{~s}, 16 \mathrm{H}), 5.22(\mathrm{~s}, 4 \mathrm{H}), 5.53$ $(\mathrm{s}, 4 \mathrm{H}), 6.59(\mathrm{~d}, 4 \mathrm{H}, J=1.8 \mathrm{~Hz}), 6.72(\mathrm{t}, 2 \mathrm{H}, J=1.8 \mathrm{~Hz}), 6.86-6.92(\mathrm{~m}, 14 \mathrm{H}), 7.05-7.10(\mathrm{~m}$, $16 \mathrm{H}), 7.14(\mathrm{~d}, 4 \mathrm{H}, J=8.7 \mathrm{~Hz}), 7.38(\mathrm{~s}, 2 \mathrm{H}), 7.63(\mathrm{~d}, 4 \mathrm{H}, J=8.7 \mathrm{~Hz}), 7.89$ (s, 4H); $8.16(\mathrm{~s}, 4 \mathrm{H})$, 8.31-8.32 (m, 4H). ${ }^{13} \mathrm{C}$ NMR (75 MHz, DMSO-d 6 ): $\delta_{\mathrm{C}} 24.9,25.1,25.9,29.3,29.4,43.9,49.1$, 49.2, 52.8, 61.2, 79.1, 101.0, 107.2, 115.2, 115.6, 122.6, 123.2, 124.3, 124.8, 126.7, 127.4, 128.4, 131.9, 132.4, 132.8, 135.6, 138.0, 142.3, 142.6, 143.3, 144.0, 159.0, 159.4, 194.9. MALDI-TOFMS: $m / z=2472.2\left(\mathrm{M}^{+}\right)$. Anal. calcd. for $\mathrm{C}_{135} \mathrm{H}_{134} \mathrm{~N}_{34} \mathrm{O}_{7} \mathrm{~S}_{4}: \mathrm{C}, 65.57 ; \mathrm{H}, 5.46 ; \mathrm{N}, 19.26 \%$. Found: C, 65.66; H, 5.54; N, 19.18\%.

Dendrimer $6\left(\mathbf{G}_{1}\right)$. Yield 86\%, mp $104{ }^{\circ} \mathrm{C} .{ }^{1} \mathrm{H}$ NMR $\left(300 \mathrm{MHz}, \mathrm{CDCl}_{3}\right): \delta_{\mathrm{H}} 1.17-1.26(\mathrm{~m}, 16 \mathrm{H})$, 1.74-1.82 (m, 16H), 2.13-2.15 (m, 2H), $2.86(\mathrm{t}, 4 \mathrm{H}, J=5.4 \mathrm{~Hz}), 4.19(\mathrm{t}, 8 \mathrm{H}, J=6.9 \mathrm{~Hz}), 4.26(\mathrm{t}$, $8 \mathrm{H}, J=6.9 \mathrm{~Hz}), 5.09(\mathrm{~s}, 8 \mathrm{H}), 5.16(\mathrm{~s}, 8 \mathrm{H}), 5.18(\mathrm{~s}, 4 \mathrm{H}), 5.41(\mathrm{~s}, 4 \mathrm{H}), 6.49(\mathrm{~d}, 4 \mathrm{H}, J=1.5 \mathrm{~Hz})$, $6.61(\mathrm{t}, 2 \mathrm{H}, J=1.8 \mathrm{~Hz}), 6.74(\mathrm{~d}, 8 \mathrm{H}, J=7.8 \mathrm{~Hz}), 6.85(\mathrm{t}, 8 \mathrm{H}, J=7.5 \mathrm{~Hz}), 6.97-7.01(\mathrm{~m}, 10 \mathrm{H})$, 7.04-7.09 (m, 10H), $7.28(\mathrm{~s}, 4 \mathrm{H}), 7.40(\mathrm{~d}, 4 \mathrm{H}, J=8.7 \mathrm{~Hz}), 7.56(\mathrm{~s}, 4 \mathrm{H}), 7.62(\mathrm{~s}, 2 \mathrm{H}), 7.69(\mathrm{~s}$, 2H). ${ }^{13} \mathrm{C} \mathrm{NMR}\left(75 \mathrm{MHz}, \mathrm{CDCl}_{3}\right): \delta_{\mathrm{C}} 22.8,25.4,25.5,28.3,29.6,29.7,30.8,44.8,49.8,49.9$, 53.9, 61.8, 101.9, 107.3, 114.6, 115.2, 122.2, 122.7, 122.8, 123.0, 123.7, 127.0, 127.2, 129.1, 132.1, 134.5, 136.1, 136.8, 143.3, 143.9, 144.1, 144.6, 158.4, 159.7, 189.9. MALDI-TOF-MS: $m / z=2484\left(\mathrm{M}^{+}\right)$. Anal. calcd. for $\mathrm{C}_{136} \mathrm{H}_{136} \mathrm{~N}_{34} \mathrm{O}_{7} \mathrm{~S}_{4}: \mathrm{C}, 65.68 ; \mathrm{H}, 5.51 ; \mathrm{N}, 19.15 \%$. Found: $\mathrm{C}$, 65.74; H, 5.64; N, 19.12\%.

Dendrimer $7\left(\mathbf{G}_{\mathbf{0}}\right)$. Yield 92\%, mp 88-90 ${ }^{\circ} \mathrm{C}$. ${ }^{1} \mathrm{H}$ NMR $\left(300 \mathrm{MHz}, \mathrm{CDCl}_{3}\right): \delta_{\mathrm{H}} 1.09-1.13(\mathrm{~m}$, 8H), 1.56-1.60 (m, 4H), 1.70-1.75 (m, 4H), 3.98 (t, 4H, J = 6.3 Hz), 4.19 (t, 4H, J = 6.9 Hz), 5.07 $(\mathrm{d}, 2 \mathrm{H}, J=12.6 \mathrm{~Hz}), 5.19(\mathrm{~s}, 4 \mathrm{H}), 5.23(\mathrm{~s}, 2 \mathrm{H}), 6.46(\mathrm{~s}, 2 \mathrm{H}), 6.76(\mathrm{~d}, 4 \mathrm{H}, J=8.1 \mathrm{~Hz}), 6.86(\mathrm{t}$, $4 \mathrm{H}, J=7.5 \mathrm{~Hz}), 7.02(\mathrm{t}, 4 \mathrm{H}, J=7.5 \mathrm{~Hz}), 7.09(\mathrm{~d}, 4 \mathrm{H}, J=6.9 \mathrm{~Hz}), 7.14-7.24(\mathrm{~m}, 4 \mathrm{H}), 7.28(\mathrm{~s}$, 2H), 7.31-7.34 (m, 2H), $7.50(\mathrm{~d}, 2 \mathrm{H}, J=9.0 \mathrm{~Hz}), 7.86(\mathrm{~d}, 2 \mathrm{H}, J=8.1 \mathrm{~Hz}), 7.93(\mathrm{~d}, 2 \mathrm{H}, J=9.0$ $\mathrm{Hz}) .{ }^{13} \mathrm{C}$ NMR $\left(75 \mathrm{MHz}, \mathrm{CDCl}_{3}\right): \delta_{\mathrm{C}} 25.6,29.7,29.8,44.9,49.8,50.0,64.0,115.3,116.0,120.7$, $122.2,122.4,122.8,123.9,124.0,125.4,126.5,127.2,127.3,127.9,129.5,129.6,133.9,144.3$, 144.6, 144.8, 153.7. ESI-MS: $m / z=1174(\mathrm{M}+\mathrm{H})^{+}$. Anal. calcd. for $\mathrm{C}_{68} \mathrm{H}_{64} \mathrm{~N}_{14} \mathrm{O}_{2} \mathrm{~S}_{2}: \mathrm{C}, 69.60 ; \mathrm{H}$, $5.50 ; \mathrm{N}, 16.71 \%$. Found: c, 69.72; H, 5.67; N, 16.78\%.

Dendrimer $8\left(\mathbf{G}_{1}\right)$. Yield 83\%, mp $98{ }^{\circ} \mathrm{C} .{ }^{1} \mathrm{H}$ NMR: $\left(300 \mathrm{MHz}, \mathrm{CDCl}_{3}\right): \delta_{\mathrm{H}} 1.20-1.29(\mathrm{~m}, 16 \mathrm{H})$, $1.75-1.77(\mathrm{~m}, 16 \mathrm{H}), 4.11-4.25(\mathrm{~m}, 16 \mathrm{H}), 4.96(\mathrm{~d}, 2 \mathrm{H}, J=12.9 \mathrm{~Hz}), 5.07(\mathrm{~s}, 12 \mathrm{H}), 5.17(\mathrm{~s}, 10 \mathrm{H})$, $6.30(\mathrm{~s}, 4 \mathrm{H}), 6.48(\mathrm{~s}, 2 \mathrm{H}), 6.62(\mathrm{~s}, 2 \mathrm{H}), 6.74(\mathrm{~d}, 8 \mathrm{H}, J=8.1 \mathrm{~Hz}), 6.86(\mathrm{t}, 8 \mathrm{H}, J=7.5 \mathrm{~Hz}), 7.02(\mathrm{t}$, $8 \mathrm{H}, J=7.5 \mathrm{~Hz}), 7.08(\mathrm{~d}, 12 \mathrm{H}, J=6.6 \mathrm{~Hz}), 7.26(\mathrm{~s}, 6 \mathrm{H}), 7.37(\mathrm{~d}, 2 \mathrm{H}, J=9.0 \mathrm{~Hz}), 7.59(\mathrm{~s}, 4 \mathrm{H})$, $7.77(\mathrm{~d}, 2 \mathrm{H}, J=8.4 \mathrm{~Hz}), 7.83(\mathrm{~d}, 2 \mathrm{H}, J=9.0 \mathrm{~Hz}) .{ }^{13} \mathrm{C} \mathrm{NMR}\left(75 \mathrm{MHz}, \mathrm{CDCl}_{3}\right): \delta_{\mathrm{C}} 25.6,25.7$, 29.8, 29.9, 44.9, 50.0, 53.7, 61.9, 63.9, 101.7, 107.3, 115.3, 116.0, 120.7, 122.4, 122.8, 123.0, 
123.8, 123.9, 125.3, 126.5, 127.2, 127.3, 128.0, 129.5, 133.8, 137.0, 143.4, 144.3, 144.7, 144.9, 153.6, 159.8. MALDI-TOF-MS: $m / z=2466(\mathrm{M})^{+}$. Anal. calcd. for $\mathrm{C}_{136} \mathrm{H}_{132} \mathrm{~N}_{34} \mathrm{O}_{6} \mathrm{~S}_{4}: \mathrm{C}, 66.21$; H, 5.39; N, 19.30\%. Found: C, 66.14; H, 5.53; N, 19.24\%.

Bromo dendron 11. Yield 90\%, mp $112{ }^{\circ} \mathrm{C} .{ }^{1} \mathrm{H}$ NMR $\left(300 \mathrm{MHz}, \mathrm{CDCl}_{3}\right): \delta_{\mathrm{H}} 1.17-1.25(\mathrm{~m}, 2 \mathrm{H})$, 1.35-1.45 (m, 2H), 1.72-1.85 (m, 4H), $3.34(\mathrm{t}, 2 \mathrm{H}, J=6.9 \mathrm{~Hz}), 4.27(\mathrm{t}, 2 \mathrm{H}, J=6.9 \mathrm{~Hz}), 5.21(\mathrm{~s}$, 2H), $6.77(\mathrm{~d}, 2 \mathrm{H}, J=8.1 \mathrm{~Hz}), 6.90(\mathrm{t}, 2 \mathrm{H}, J=7.5 \mathrm{~Hz}), 7.02-7.08(\mathrm{~m}, 2 \mathrm{H}), 7.11-7.14(\mathrm{~m}, 2 \mathrm{H})$, $7.28(\mathrm{~s}, 1 \mathrm{H}) .{ }^{13} \mathrm{C}$ NMR $\left(75 \mathrm{MHz}, \mathrm{CDCl}_{3}\right): \delta_{\mathrm{C}} 25.4,27.4,29.9,32.3,33.4,45.0,50.2,115.3$, 122.3, 122.8, 123.9, 127.2, 127.3, 144.3, 144.8. ESI-MS: $m / z=444(\mathrm{M}+\mathrm{H})^{+}$. Anal. calcd. for $\mathrm{C}_{21} \mathrm{H}_{23} \mathrm{BrN}_{4} \mathrm{~S}: \mathrm{C}, 56.88 ; \mathrm{H}, 5.23 ; \mathrm{N}, 12.64 \%$. Found: $\mathrm{C}, 56.72 ; \mathrm{H}, 5.46 ; \mathrm{N}, 12.48 \%$.

Dendritic chloride 14. Yield 89\%, mp 218-220 ${ }^{\circ} \mathrm{C}$. ${ }^{1} \mathrm{H}$ NMR $\left(300 \mathrm{MHz}, \mathrm{CDCl}_{3}\right)$ : $\delta_{\mathrm{H}} 1.19-1.29$ $(\mathrm{m}, 8 \mathrm{H}), 1.78-1.84(\mathrm{~m}, 8 \mathrm{H}), 4.23(\mathrm{t}, 4 \mathrm{H}, J=7.2 \mathrm{~Hz}), 4.28(\mathrm{t}, 4 \mathrm{H}, J=7.2 \mathrm{~Hz}), 4.48(\mathrm{~s}, 2 \mathrm{H}), 5.16$ (s, 4H), $5.19(\mathrm{~s}, 4 \mathrm{H}), 6.59$ (t, 1H, $J=2.1 \mathrm{~Hz}), 6.63(\mathrm{~d}, 2 \mathrm{H}, J=2.1 \mathrm{~Hz}), 6.76(\mathrm{~d}, 4 \mathrm{H}, J=8.1 \mathrm{~Hz})$, 6.85-6.90 (m, 4H), 7.01-7.12 (m, 8H), 7.28 (s, 2H), $7.56(\mathrm{~s}, 2 \mathrm{H}) .{ }^{13} \mathrm{C} \mathrm{NMR}\left(75 \mathrm{MHz}, \mathrm{CDCl}_{3}\right): \delta-$ с 25.6, 29.9, 41.7, 44.9, 49.4, 49.6, 50.0, 54.3, 61.8, 107.2, 115.3, 122.2, 122.6, 122.8, 123.8, 123.4, 127.1, 127.4, 143.4, 143.6, 144.2, 144.7, 159.1. ESI-MS: $m / z=1046.7(\mathrm{M}+\mathrm{H})^{+}$. Anal. calcd. for $\mathrm{C}_{55} \mathrm{H}_{57} \mathrm{ClN}_{14} \mathrm{O}_{2} \mathrm{~S}_{2}$ : C, 63.17; H, 5.49; N, 18.75\%. Found: C, 63.29; H, 5.58; N, $18.69 \%$.

General procedure for the synthesis of dendritic azide from the dendritic chloride/bromide To the corresponding alkyl chloride/bromide $(1 \mathrm{mmol})$ dissolved in dry DMF $(20 \mathrm{ml})$, sodium azide $(1.5 \mathrm{mmol})$ was added and stirring was continued at $60{ }^{\circ} \mathrm{C}$ for $10 \mathrm{~h}$. The reaction mixture was then allowed to cool to room temperature. It was then poured into ice-cold water $(30 \mathrm{~mL})$ and extracted with $\mathrm{CHCl}_{3}(3 \times 100 \mathrm{~mL})$. The organic layer was washed with water $(100 \mathrm{~mL})$ and saturated $\mathrm{NaCl}(3 \times 100 \mathrm{~mL})$, dried $\left(\mathrm{Na}_{2} \mathrm{SO}_{4}\right)$. Solvent was evaporated under reduced pressure to afford the crude product, which was purified by column chromatography $\left(\mathrm{SiO}_{2}\right)$.

Azido dendron 12. Yield $91 \%$, mp 84-86 ${ }^{\circ} \mathrm{C} .{ }^{1} \mathrm{H}$ NMR $\left(300 \mathrm{MHz}, \mathrm{CDCl}_{3}\right): \delta_{\mathrm{H}} 1.17-1.36(\mathrm{~m}$, $4 \mathrm{H}), 1.47-1.54(\mathrm{~m}, 2 \mathrm{H}), 1.77-1.85(\mathrm{~m}, 2 \mathrm{H}), 3.21(\mathrm{t}, 2 \mathrm{H}, J=6.9 \mathrm{~Hz}), 4.26(\mathrm{t}, 2 \mathrm{H}, J=6.9 \mathrm{~Hz}), 5.21$ (s, 2H), $6.76(\mathrm{~d}, 2 \mathrm{H}, J=8.1 \mathrm{~Hz}), 6.90(\mathrm{t}, 2 \mathrm{H}, J=7.5 \mathrm{~Hz}), 7.02-7.05(\mathrm{~m}, 2 \mathrm{H}), 7.08-7.14(\mathrm{~m}, 2 \mathrm{H})$, $7.28(\mathrm{~s}, 1 \mathrm{H}) .{ }^{13} \mathrm{C}$ NMR $\left(75 \mathrm{MHz}, \mathrm{CDCl}_{3}\right): \delta_{\mathrm{C}} 25.8,26.0,28.6,29.9,45.0,50.2,51.2,115.3$, 122.3, 122.8, 123.9, 127.2, 127.3, 144.3, 144.8. ESI-MS: $m / z=406(\mathrm{M}+\mathrm{H})^{+}$. Anal. calcd. for $\mathrm{C}_{21} \mathrm{H}_{23} \mathrm{~N}_{7} \mathrm{~S}: \mathrm{C}, 62.20 ; \mathrm{H}, 5.72 ; \mathrm{N}, 24.18 \%$. Found: C, 62.04; H, 4.87; N, 24.09\%.

Dendritic azide 15. Yield 87\%, mp $106{ }^{\circ} \mathrm{C} .{ }^{1} \mathrm{H}$ NMR $\left(300 \mathrm{MHz}, \mathrm{CDCl}_{3}\right)$ : $\delta_{\mathrm{H}} 1.11-1.18(\mathrm{~m}, 8 \mathrm{H})$, 1.56-1.85 (m, 8H), 4.11-4.21 (m, 10H), $5.08(\mathrm{~s}, 4 \mathrm{H}), 5.10(\mathrm{~s}, 4 \mathrm{H}), 6.47$ (d, $2 \mathrm{H}, J=1.8 \mathrm{~Hz}), 6.53$ $(\mathrm{t}, 1 \mathrm{H}, J=2.1 \mathrm{~Hz}), 6.67(\mathrm{~d}, 4 \mathrm{H}, J=8.1 \mathrm{~Hz}), 6.79(\mathrm{t}, 4 \mathrm{H}, J=7.2 \mathrm{~Hz}), 6.94(\mathrm{t}, 4 \mathrm{H}, J=7.5 \mathrm{~Hz})$, $7.01(\mathrm{~d}, 4 \mathrm{H}, J=7.5 \mathrm{~Hz}), 7.19(\mathrm{~s}, 2 \mathrm{H}), 7.49$ (s, $2 \mathrm{H}) .{ }^{13} \mathrm{C} \mathrm{NMR}\left(75 \mathrm{MHz}, \mathrm{CDCl}_{3}\right): \delta_{\mathrm{C}} 25.4,25.6$, 29.7, 29.8, 44.8, 49.8, 49.9, 54.5, 61.9, 101.6, 107.4, 115.1, 122.2, 122.6, 122.7, 123.7, 127.0, 127.2, 137.7, 143.6, 144.1, 144.6, 159.5. ESI-MS: $m / z=1052(\mathrm{M}+\mathrm{H})^{+}$. Anal. calcd. for $\mathrm{C}_{55} \mathrm{H}_{57} \mathrm{~N}_{17} \mathrm{O}_{2} \mathrm{~S}_{2}$ : C, 62.78; H, 5.46; N, 22.63\%. Found: C, 63.69; H, 5.52; N, 22.69\%.

General procedure for synthesis of acetylenic enone core. A mixture of the corresponding phenol (1.0 equiv.), propargyl bromide (1.25 equiv.) and anhydrous potassium carbonate (3.0 
equiv.) in dry DMF (15 mL) was stirred at $60{ }^{\circ} \mathrm{C}$ for $24 \mathrm{~h}$. The reaction mixture was then allowed to cool to room temperature and poured into ice water. The resulting precipitate was filtered, washed thoroughly with water and dissolved in $\mathrm{CHCl}_{3}(150 \mathrm{~mL})$. The organic layer was washed with water $(2 \times 100 \mathrm{~mL})$ and brine $(1 \times 150 \mathrm{~mL})$, dried $\left(\mathrm{Na}_{2} \mathrm{SO}_{4}\right)$ and evaporated to give the crude dendron, which was purified by column chromatography $\left(\mathrm{SiO}_{2}\right)$.

Bis(propargyloxy)dienone 19. Yield 63\%, mp $83{ }^{\circ} \mathrm{C} .{ }^{1} \mathrm{H} \mathrm{NMR}\left(300 \mathrm{MHz}, \mathrm{CDCl}_{3}\right): \delta_{\mathrm{H}} 2.56(\mathrm{t}$, $2 \mathrm{H}, J=2.1 \mathrm{~Hz}), 4.74(\mathrm{~d}, 4 \mathrm{H}, J=2.1 \mathrm{~Hz}), 6.93(\mathrm{~s}, 2 \mathrm{H}), 7.01(\mathrm{~d}, 4 \mathrm{H}, J=9.0 \mathrm{~Hz}), 7.58(\mathrm{~d}, 4 \mathrm{H}, J=$ $8.7 \mathrm{~Hz}), 7.69(\mathrm{~d}, 2 \mathrm{H}, J=15.0 \mathrm{~Hz}) .{ }^{13} \mathrm{C} \mathrm{NMR}\left(75 \mathrm{MHz}, \mathrm{CDCl}_{3}\right): \delta_{\mathrm{C}} 55.9,76.0,78.6,114.9,115.3$, 123.9, 128.5, 130.0, 142.5, 159.4, 188.8. ESI-MS: $m / z=343(\mathrm{M}+\mathrm{H})^{+}$. Anal. calcd. for $\mathrm{C}_{23} \mathrm{H}_{18} \mathrm{O}_{3}$ : C, 80.68; H, 5.30\%. Found: C, 80.56; H, 5.38\%.

Bis(propargyloxy)pentanone 20. Yield $82 \%$, mp $172{ }^{\circ} \mathrm{C} .{ }^{1} \mathrm{H}$ NMR $\left(300 \mathrm{MHz}, \mathrm{CDCl}_{3}\right): \delta_{\mathrm{H}} 2.56$ $(\mathrm{t}, 2 \mathrm{H}, J=2.4 \mathrm{~Hz}), 3.09(\mathrm{~s}, 4 \mathrm{H}), 4.75(\mathrm{~d}, 4 \mathrm{H}, J=2.4 \mathrm{~Hz}), 7.05(\mathrm{~d}, 4 \mathrm{H}, J=8.7 \mathrm{~Hz}), 7.56-7.60$ (m, $6 \mathrm{H}) .{ }^{13} \mathrm{C}$ NMR $\left(75 \mathrm{MHz}, \mathrm{CDCl}_{3}\right): \delta_{\mathrm{C}} 26.4,55.8,75.9,78.1,115.2,129.6,132.4,133.1,135.7$, 158.4, 196.2. ESI-MS: $m / z=369(\mathrm{M}+\mathrm{H})^{+}$. Anal. calcd. for $\mathrm{C}_{25} \mathrm{H}_{20} \mathrm{O}_{3}: \mathrm{C}, 81.50 ; \mathrm{H}, 5.47 \%$. Found: C, 81.58; H, 5.52\%.

Bis(propargyloxy)hexanone 21. Yield 76\%, mp 152-154 ${ }^{\circ} \mathrm{C} .{ }^{1} \mathrm{H}$ NMR $\left(300 \mathrm{MHz}, \mathrm{CDCl}_{3}\right): \delta_{\mathrm{H}}$ $1.79(\mathrm{~m}, 2 \mathrm{H}), 2.55(\mathrm{t}, 2 \mathrm{H} . J=2.1 \mathrm{~Hz}), 2.91(\mathrm{t}, 4 \mathrm{H}, J=5.4 \mathrm{~Hz}), 4.73(\mathrm{~d}, 4 \mathrm{H}, J=2.1 \mathrm{~Hz}), 7.51(\mathrm{~d}$, $4 \mathrm{H}, J=8.7 \mathrm{~Hz}), 7.45(\mathrm{~d}, 4 \mathrm{H}, J=8.7 \mathrm{~Hz}), 7.75(\mathrm{~s}, 2 \mathrm{H}) .{ }^{13} \mathrm{C} \mathrm{NMR}\left(75 \mathrm{MHz}, \mathrm{CDCl}_{3}\right): \delta_{\mathrm{C}} 23.0$, 28.5, 55.8, 75.9, 78.2, 116.2, 128.5, 129.6, 134.7, 136.4, 157.7, 190.2. ESI-MS: $\mathrm{m} / z=383$ $(\mathrm{M}+\mathrm{H})^{+}$. Anal. calcd. for $\mathrm{C}_{26} \mathrm{H}_{22} \mathrm{O}_{3}: \mathrm{C}, 81.65 ; \mathrm{H}, 5.80 \%$. Found: C, 81.58; H, 5.86\%.

\section{Supplementary Material}

${ }^{1} \mathrm{H},{ }^{13} \mathrm{C}$ NMR and Mass spectra for dendrimer $\mathbf{2}$ and $\mathbf{4}$ are available.

\section{Acknowledgements}

The authors thank DST-FIST for providing NMR facilities to the Department of Organic Chemistry, University of Madras. CSK thank CSIR for the award of Senior Research Fellowship (SRF).

\section{References}

1. Lo, S. C.; Burn, P. L. Chem. Rev. 2007, 107, 1097. http://dx.doi.org/10.1021/cr0501361 PMid:17385927

2. Calabretta, M. K.; Kumar, A.; McDermott, A. M.; Cai, C. Biomacromolecules 2007, 8, 1807. http://dx.doi.org/10.1021/bm0701088 
PMid:17511499

PMCid:PMC2430505

3. Svenson, S.; Tomalia, D. A. Adv. Drug Delivery Rev. 2005, 57, 2106.

http://dx.doi.org/10.1016/j.addr.2005.09.018 PMid:16305813

4. Kwon, T. H.; Kim, M. K.; Kwon, J.; Shin, D. J.; Park, S. J.; Lee, C. L.; Kim, J. J.; Hong, J. I. Chem. Mater. 2007, 19, 3673.

http://dx.doi.org/10.1021/cm070536k

5. Wu, W.; Li, C.; Yu, G.; Liu, Y.; Ye, C.; Qin, J.; Li, Z. Chem.--Eur. J. 2012, 18, 11019. http://dx.doi.org/10.1002/chem.201200441 PMid:22829501

6. Hwang, S. H.; Moorefield, C. N.; Newkome, G. R. Chem. Soc. Rev. 2008, 37, 2543. http://dx.doi.org/10.1039/b803932c

PMid:18949125

7. Schlupp, M.; Weil, T.; Berresheim, A. J.; Wiesler, U. M.; Bargon, J.; Mullen, K. Angew. Chem., Int. Ed. 2001, 40, 4011. http://dx.doi.org/10.1002/1521-3773(20011105)40:21<4011::AID-ANIE4011>3.0.CO;2-C

8. Rostovtsev, V. V.; Green, L. G.; Fokin, V. V.; Sharpless, K. B. Angew. Chem. Int. Ed. 2002, $41,2596$.

http://dx.doi.org/10.1002/1521-3773(20020715)41:14<2596::AID-ANIE2596>3.0.CO;2-4

9. Alvarez, R.; Velazquez, S.; San, F.; Aquaro, S.; De, C.; Perno, C. F.; Karlsson, A.;Balzarini, J.; Camarasa, M. J. J. Med. Chem. 1994, 37, 4185.

http://dx.doi.org/10.1021/jm00050a015

10. Chen, X. M.; Li, Z. J.; Ren, Z. X.; Huang, Z. T. Carbohydr. Res. 1999, 315, 262. http://dx.doi.org/10.1016/S0008-6215(99)00020-8

11. Genin, M. J.; Allwine, D. A.; Anderson, D. J.; Barbachyn, M. R.; Emmert, D. E.; Garmon, S. A.; Graber, D. R.; Grega, K. C.; Hester, J. B.; Hutchinson, D. K.; Morris, J.; Reischer, R. J.; Ford, C. W.; Zurenko, G. E.; Hamel, J. C.; Schaadt, R. D.; Stapert, D.; Yagi, B. H. J. Med. Chem. 2000, 43, 953.

http://dx.doi.org/10.1021/jm990373e

12. Gopin, A.; Ebner, S.; Attali, B.; Shabat, D. Bioconjugate Chem. 2006, 17, 1433.

http://dx.doi.org/10.1021/bc060180n

PMid:17105221

13. Thomas, T. P.; Huang, B.; Choi, S. K.; Silpe, J. E.; Kotlyar, A.; Desai, A. M.; Zong, H.; Gam, J.; Joice, M.; Baker, J. J. R. Mol. Pharmaceutics 2012, 9, 2669.

http://dx.doi.org/10.1021/mp3002232

PMid:22827500

14. Rajakumar, P.; Raja, S.; Satheeshkumar, C.; Ganesan, S.; Maruthamuthu, P.; Suthanthiraraj, S. A. New J. Chem., 2010, 34, 2247.

http://dx.doi.org/10.1039/c0nj00044b

15. Rajakumar, P.; Raja, S.; Thirunarayanan, A. Synlett 2010, 11, 1669.

http://dx.doi.org/10.1055/s-0030-1258082 
16. Rajakumar, P.; Raja, R. Tetrahedron Lett. 2010, 51, 4365.

http://dx.doi.org/10.1016/j.tetlet.2010.06.059

17. Rajakumar, P.; Visalakshi, K.; Ganesan, S. Maruthamuthu, P.; Suthanthiraraj, S. A. J. Mater. Sci. 2012, 47, 1811. http://dx.doi.org/10.1007/s10853-011-5967-9

18. Kim, G. W.; Cho, M. J.; Yu, Y. J.; Kim, Z. H.; Jin, J. I.; Kim, D. Y.; Choi, D. H. Chem. Mater. 2007, 19, 42.

http://dx.doi.org/10.1021/cm0618493

19. Pluta, K.; MIodawska, B. M.; Jelen, M. Eur. J. Med. Chem. 2011, 46, 3179. http://dx.doi.org/10.1016/j.ejmech.2011.05.013

PMid:21620536

20. Ma, L.; Lee, S. J.; Lin, W. Macromolecules 2002, 35, 6178. http://dx.doi.org/10.1021/ma020283a

21. Liu, G.-H.; Fan, Q.-H.; Yang, X.-Q.; Chen, X.-M. Arkivoc 2003, (ii), 123. http://dx.doi.org/10.3998/ark.5550190.0004.214

22. Sae-Lim, C.; Sandman, D. J.; Foxman, B. M.; Sukwattanasinitt, M. J. Macromol. Sci., Part A: Pure Appl. Chem. 2006, 43, 1929. http://dx.doi.org/10.1080/10601320600996114

23. Du, Z. Y.; Liu, R. R.; Shao, W.Y.; Mao, X. P.; Ma, L.; Gu, L. Q.; Huang, Z. S.; Chan, A. S. C. Eur. J. Med. Chem. 2006, 41, 213. http://dx.doi.org/10.1016/j.ejmech.2005.10.012

PMid:16387392 